\title{
A FOUR-QUADRANT FLOATING-GATE SYNAPSE
}

\author{
Paul Hasler, Chris Diorio, and Bradley A. Minch \\ Georgia Institute of Technology \\ Atlanta, GA 30332-0250 \\ phasler@ee.gatech.edu
}

\begin{abstract}
We present a new type of pFET synapse; by degenerating the source, the oxide currents provide stabilizing feedback to the floating gate and to the drain. We present experimental measurements from a floating-gate synapse that simultaniously computes four-quadrant products of its input and weight values, and computes a four-quadrant correlation, typical of hebbian and backpropagation learning rules, between the input and drain voltages. Our four-quadrant synapse is built from two source-degenerated pFET synapses; by adding weak exponential feedback to the source of a floating-gate $\mathrm{pFET}$ synapse, we obtain a floating-gate synapse with unique dynamical properties. This four-quadrant synapse can become the fundamental building block of many continuoustime neural-network learning algorithms.
\end{abstract}

Floating-gate devices have come to be seen not only as memory elements, but as continuous-time circuit elements operating a variety of timescales. This paper presents a floating-gate synapse that computes four-quadrant products of its input $\left(X_{i}\right)$ and its weight value $\left(W_{i, j}\right)$, and computes a four-quadrant correlation of the input and error signal $\left(e_{i}\right)$. Figure 1 shows a diagram of a continuously adapting neural array; the inset shows schematically the synapse computations for the $i_{t h}$ row and for the $j_{t h}$ column. This error signal could either be a function of the output, as in Hebbian or other unsupervised algorithms, or due to an error in the desired output, as in gradient-descent algorithms. Four-quadrant multiplications are essential in most adaptive-filter and neural-network applications. Biological synapses use two-quadrant multiplications, but excitatory (positive multiplication) and inhibitory (negative multiplication) synapses are used in a cooperative fashion to achieve this functionality when needed [3].

Our four-quadrant floating-gate synapses, as well as our previous synapses [2], are well suited to large synaptic arrays because of five important properties. First, in the absence of learning, the weight is stored permanently, because the charge leakage from a floating gate is negligible. Second, the synapse's output current is the four-quadrant product of the input signal with the synaptic weight. Third, because our synapse only requires two floating-gate transistors per synapse, the synapse still requires minimal area. Fourth, the synapse can dissipate a minimal amount of power, because it can operate at subthreshold current levels. Fifth, this synapse implements a four-quadrant learning rule for

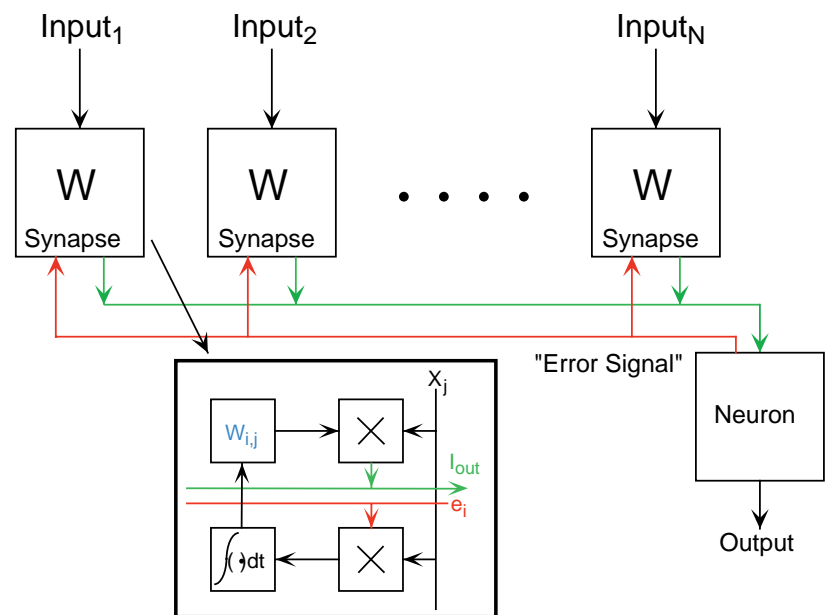

Figure 1: Schematic diagram of a continuously-adapting neural network. The neuron block cuold span the range from a sinple transresistance element to a biologically inspired neuron model. Inset: Block diagram of a synapse at the $i^{t h}$ row and at the $j_{t h}$ column in this neural array. A continuous-time synapse simultaniously outputs the product of its input $\left(X_{j}\right)$ and its weight value $\left(W_{i, j}\right)$, as well as updating the weight value based on the product of its input and the broadcasted error signal $\left(e_{i}\right)$. Ideally, we want the multiplications to be four-quadrant multiplies.

modifying the weight on the floating gate; the form of this rule depends on how various error signals are fed back to the floating gate.

\section{SOURCE-DEGENERATED PFET SYNAPSE}

Figure 2 shows the circuit model for the source-degenerated (s-d) $p$ FET synapse; we presented the initial results previously [2]. Previous nFET or pFET synapses provided only either stabilizing feedback to the floating gate or stabilizing feedback to the drain; the gate currents of this s-d pFET synapse provide stabilizing feedback to both the floating gate and to the drain, making it a practical candidate for continuously operating arrays of floating-gate synapses. We will assume that all the floating-gate devices are matched. We define $C_{T}$ as the total amount of capacitance connected 


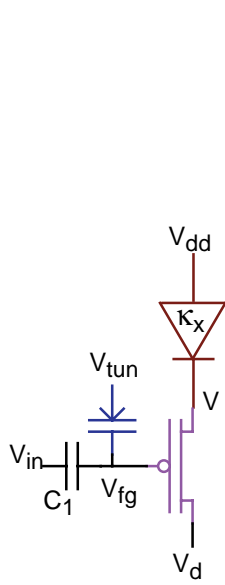

(a)

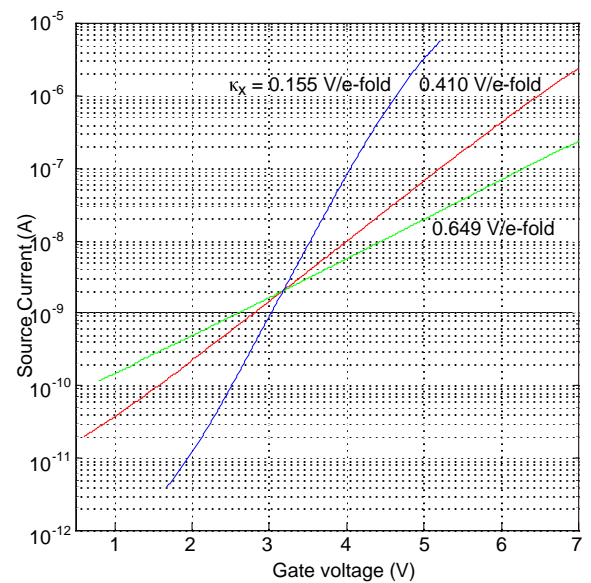

(b)

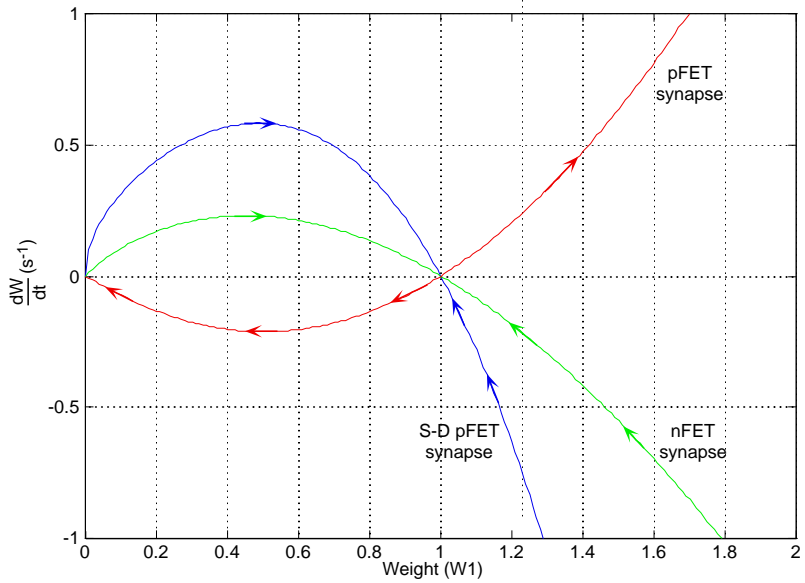

(c)

Figure 2: The source-degenerated (s-d) $p$ FET synapse. (a) Circuit diagram of the s-d $p$ FET synapse. The s-d $p$ FET synapse is comprised of a $p$ FET single-transistor synapse and the $\kappa_{x}$ diode element, which provides feedback to the source terminal. This feedback results in a weaker exponential current dependance of the gate voltage, and changes the circuit dynamics resulting from the floating-gate currents. We utilize the DIBL effect in short-channel MOSFETs to build a compact $\kappa_{x}$ element. (b) Drain current versus gate voltage for two different values of $\kappa_{x}$, as well as for no $\kappa_{x}$ element. (c) Plot of $\frac{d W}{d t}$ versus $W$ for different values of $V_{t u n}$ and $V_{d d}$. The weight of the s-d pFET synapse, as well as the nFET synapse, tends to converge towards 1 , where the weight of the pFET synapse tends to diverge from 1.

to the floating gate, and $C_{2}$ as the capacitance between floating gate and drain (which is not explicitly drawn for clarity).

We model the $\kappa_{x}$-diode element, seen in Fig. 2, as a weakly exponential device:

$$
I=I_{x} e^{-\kappa_{x} \Delta V / U_{T}},
$$

where $\Delta V$ represents the change in $V$ from the bias level, $I_{x}$ is the quiesent current through the device, and $\kappa_{x}$ is a device parameter. We build integrated synapses using a short-channel $p \mathrm{FET}$ with significant drain-induced barrier lowering (DIBL) to implement the $\kappa_{x}$ diode-element. A transistor that strongly exhibits DIBL shows an exponential change in current for a linear change in drain voltage.

For this paper, we consider the single-transistor synapse operating with subthreshold channel currents; many of the behaviors extend qualitatively to above-threshold operation. We will model voltage and current swings around the circuit's steady-state values, because the input signals are capacitively coupled to the floating gate. By equating the drain current in a subthreshold $p$ FET to the $\kappa_{x}$-diode current, we obtain the source-degenerated $p$ FET model equation for $\kappa_{x}$ much less than 1, which is our region of interest. We describe the subthreshold floating-gate s-d pFET channel current in saturation, $I_{s}$, for a change in the pFET's floating-gate voltage, $\Delta V_{f g}$, around a bias current, $I_{s o}$, as (neglecting Early effect) [1]

$$
I_{s}=I_{s o} e^{-\kappa_{x} \kappa_{p} \Delta V_{f g} / U_{T}}
$$

where $\kappa_{p}$ is the fractional change in the $p$ FET surface potential due to a change in $\Delta V_{f g}$, and $U_{T}$ is the thermal voltage, $\frac{k T}{q}$. Figure $2 \mathrm{~b}$ shows the channel current through this synapse as a function of gate voltage for three sizes of $\kappa_{x}$; decreasing $\kappa_{x}$ reduces the change in channel current for a fixed gate-voltage swing. The $\kappa_{x}$ element is effectively cascoded by the $p$ FET; therefore, the maximum voltage gain of this transistor is is identical to that of a subthreshold $p$ FET transistor.

To analyze the adaptation behavior in FGMOS circuits, we decompose our variables into components that change at fast and slow rates. The fast-rate variables represent the rapid changes due to the input signals; the slow-rate variables represent the floating-gate charge. We represent the weight, $W$, as the slow change in the source current due to changes in the floating-gate charge. We can modify (2) in terms of these fast and slow variables as

$$
I_{s}=I_{s o} W \exp \left(-\frac{C_{1}}{C_{T}} \frac{\kappa_{x} \kappa_{p}}{U_{T}} \Delta V_{i n}\right)
$$

$\Delta V_{f g}$ is an attenuated factor of $\Delta V_{i n}$, because we capacitively couple into the floating-gate.

We use hot-electron injection to add electrons to floating gates, and we use electron tunneling to remove electrons from floating gates. We showed previously that we can model the floating-gate current, the difference between the tunneling current and the injection current, for the sourcedegenerated pFET synapse as [2]

$$
I_{t u n}-I_{i n j}=I_{t u n 0}\left(e^{-\Delta V_{f g} / V_{x}}-e^{\sigma \Delta V_{f g} / U_{T}} e^{-\Delta V_{d} / V_{i n j}}\right),
$$

where $I_{t u n 0}$ is the quiescent tunneling current, $V_{x}$ is a tunneling device parameter related to the quiescent tunneling and floating-gate voltages, $V_{i n j}$ is an injection device 


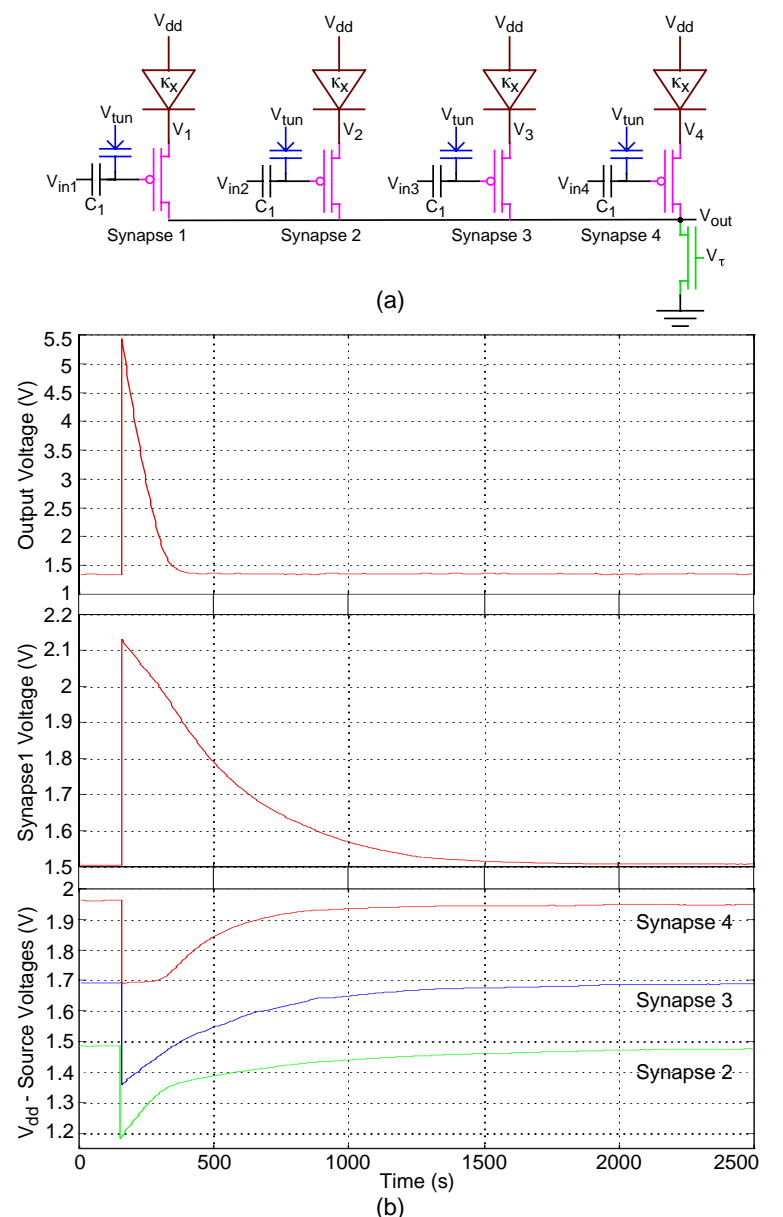

(b)

Figure 3: Circuit of the four-input source-degenerated pFET synapse. This figure shows that the stabilizing behavior for two synapses is extendible to multiple synapses. (a) Circuit diagram. (b) Output voltage and synapse voltage $\left(V_{1}, V_{2}, V_{3}, V_{4}\right)$ responses due to an input step applied to the first synapse.

parameter related to the pFET's drain voltage, and $\sigma=$ $\kappa_{p}\left(\frac{U_{T}}{V_{i n j}}-\kappa_{x} \alpha\right)$, for small $\kappa_{x}$. Our experimental measurements of a s-d pFET show that (4), which uses linearized approximations in the exponentials, accurately models the tunneling current over a wide range of channel currents, and accurately models the injection current over an order of magnitude channel currents, as well as over $1 \mathrm{~V}$ change in the drain voltage.

For a constant $V_{i n}$ and $V_{d}$, we equate the currents at the floating gate:

$$
C_{T} \frac{d V_{f g}}{d t}=-\frac{C_{T} U_{T}}{\kappa_{p} \kappa_{x}} \frac{d W}{d t}=I_{t u n}-I_{i n j}
$$

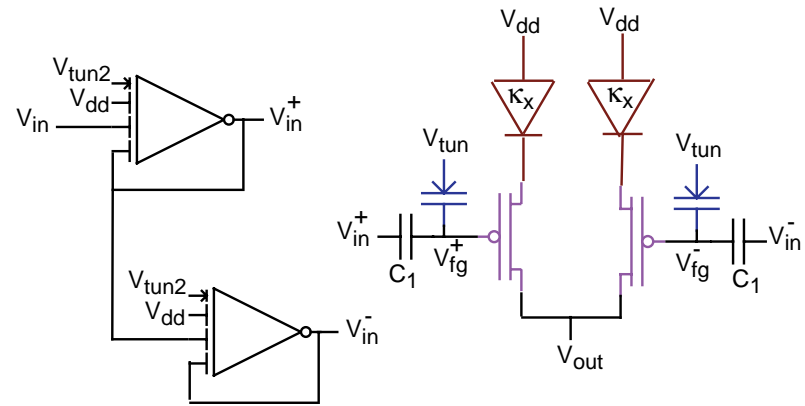

(a)

(b)

Figure 4: Circuit of the four-quadrant floating-gate synapse. (a) We use two AFGAs to generate $V_{i n}^{+}$and $V_{i n}^{-}$for a column of synapses; using two AFGAs per column has little effect on chip area of the synaptic array. The AFGAs filter slowly varying input signals and provide an inverted signal to the complementary synapse element. (b) This synapse is built from two s-d pFET synapses. Using complementary inputs results in an output current that is a four-quadrant multiplication of this input and the stored weight.

resulting in the following dynamical equation

$$
\begin{aligned}
\tau \frac{d W}{d t} & =\left(\frac{I_{s}}{I_{s o}}\right)^{1-\frac{\sigma}{\kappa_{p} \kappa_{x}}} e^{-\Delta V_{d} / V_{i n j}}\left(\frac{I_{s}}{I_{s o}}\right)^{1+\frac{U_{T}}{\kappa_{p} \kappa_{x} V_{x}}} \\
& =W^{1-\frac{\sigma}{\kappa_{p} \kappa_{x}}} e^{-\Delta V_{d} / V_{i n j}}-W^{1+\frac{U_{T}}{\kappa_{p} \kappa_{x} V_{x}}}
\end{aligned}
$$

where $\tau=C_{T} U_{T} /\left(\kappa_{p} \kappa_{x} I_{\text {tun } 0}\right)$. Figure $2 \mathrm{c}$ illustrates the dynamics of (4) by plotting the derivative of $W$ versus $W$. From Fig. 2c we see that the s-d pFET synapse tends to converge to its steady state at $W=1$; if $W>1$, the negative derivative decreases $\mathrm{W}$ towards 1 , else if $W<1$, the positive derivative increases $\mathrm{W}$ towards 1 . The trajectories for the basic pFET synapse diverge from 1. Adding the source-degeneration feedback resulted in a stablizing pFET synapse, because for small $\kappa_{x}$, an increase in the source current requires a large increase in the source voltage, which decreases the drain-to-source voltage, and that, in turn decreases the injection current.

\section{COUPLED ARRAYS OF S-D PFET SYNAPSES}

A practical application is to couple an array of synapses, such that each shares the same drain voltage, and the total current from this drain line is fixed by a transistor current source. Figure 3 shows experimental measurements of four s-d pFET transistors coupled through their drain terminals. We can indirectly measure the synapse currents by measuring the source voltages $\left(V_{1}, V_{2}, V_{3}\right.$, and $\left.V_{4}\right)$, because the current through the $\kappa_{x}$ elements is a known exponential function of these source voltages. We first see that the output voltage and the individual source currents have settled to non-zero steady-states, which would only occur if the gate-current feedback was stabilizing in both changes in the gate voltage and in the drain voltage. We then see that after applying a voltage step to the first input, the 


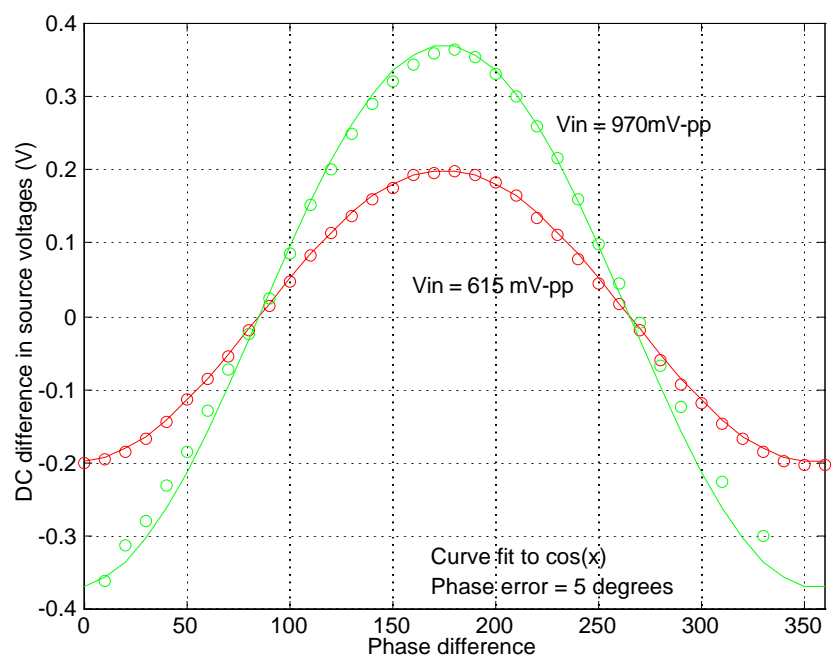

Figure 5: Difference of the DC source voltages due to two $220 \mathrm{~Hz}$ sine-wave inputs of versus phase difference between the two sine waves. In the first case, both input and output amplitudes were $615 \mathrm{mV}$-pp, and in the second case, both input and output amplitudes were $970 \mathrm{mV}$-pp. DC level of the drain voltage was $1.778 \mathrm{~V}$, and $V_{d d}$ was $12 \mathrm{~V}$. We curve fitted this output to a cosine function with 5 degrees of phase. This measurement shows that the circuit accurately encodes the positive and negative correlation between the two sine waves.

output voltage, as well as the synapse currents, returns to the equilibrium values. The two s- $\mathrm{d} p \mathrm{FET}$ synapses cooperate for the entire available channel current; regardless of the starting position, the two synapses converge to nearly equal channel currents. Both original nFET and pFET synapses have unstable properties for this experiment [2]; a coupled network of $p$ FET synapses will have at most one non-zero weight for constant inputs.

\section{A FOUR-QUADRANT FLOATING-GATE SYNAPSE}

Figure 4 shows the circuit and symbol from the floatinggate synapse. The input to this synapse, or a column of synapses, enters in on AFGAs (Autozeroing Floating-Gate Amplifiers, see $[2,1])$ to eliminate any slow variation on the input signal and to provide an inverted version of the input. This synapse couples two s-d $p$ FET synapses in a way that subtracts out their common responses to achieve a fourquadrant multiplication and correlation. The dynamics of two or more coupled floating-gate synapses result in one to all synapses settling to a non-zero weight [2].

We will first show that this simple modification naturally gives us four quadrant multiplications. The output (drain) current is the sum of the current from each s-d pFET transistor:

$I_{\text {out }}=I_{\text {so }}\left(W^{+} \exp \left(-\frac{\kappa \kappa_{x} C_{1} \Delta V_{\text {in }}}{C_{T} U_{T}}\right)+W^{-} \exp \left(\frac{\kappa \kappa_{x} C_{1} \Delta V_{\text {in }}}{C_{T} U_{T}}\right)\right) ;$

assuming the inputs are within the input linear range, $U_{T} /\left(\kappa_{x} \kappa\right)$, then we approximate the exponentials as linear functions:

$$
I_{\text {out }} \approx I_{\text {so }}\left(W^{+}+W^{-}\right)+\frac{\kappa \kappa_{x} C_{1} I_{s o}}{C_{T} U_{T}}\left(W^{+}-W^{-}\right) \Delta V_{i n},
$$

where $W^{+}$and $W^{-}$are the synapses corresponding to $V_{i n}^{+}$ and $V_{i n}^{-}$, respectively. The synapse weight, defined by $W^{+}{ }_{-}^{i n}$ $W^{-}$, and $\Delta V_{\text {in }}$ take on both positive and negative values; therefore the change in the output current is a fourquadrant product of the input by the synapse weight for fast timescales. the first term is constant for fast signals, and is typically part of the biasing circuitry. This part of the circuit is simlar to other four-quadrant multipliers used in continuous-time filters $[4,5]$.

The difficult part is to get simultanious four-quadrant correlations in the same circuit configuration. Consider the synapse in Fig. 4 with inputs applied to $V_{\text {in }}$ and $V_{\text {out }}$ around a constant bias voltage. We can write the dynamics for the two weights, $W^{+}$and $W^{-}$, from (4) by substituting the appropriate variables. We can expand these two equation2 around $\Delta V_{\text {in }}=0, \Delta V_{\text {out }}=0$, and $W=1$ upto second order, and then average over many periods of the input signal. The resulting approximate steady-state solution for $W^{+}-W^{-}$for these two differential equations is

$$
W^{+}-W^{-}=-\frac{C_{1}}{C_{T}} \frac{E\left[\Delta V_{\text {in }} \Delta V_{\text {out }}\right]}{2 V_{\text {inj } j} \frac{U_{T}}{\kappa_{x}}\left(1+\frac{V_{i n j}}{V_{x}}\right)}
$$

where $\Delta V_{\text {in }}$, and $\Delta V_{\text {out }}$ are either positive or negative, and we use $E[\cdot]$ to represent an average of the arguement over many periods of the input signal.

For two phase shifted sinusoidial inputs at the input and output terminals, the resulting correlation will be the cosine of the phase shift between the two signals. The results of this experiment is shown in Fig. 5; we measured the steady-state channel currents to obtain the difference of the weights. Qualitatively, we immediately see that we get a four-quadrant correlation, because a 180 degree phase shift in one input inverts the sign of the weight. We get similar results for two phase-shifted square-wave inputs. The measured data closely matches the fit to a cosine function with an additional phase shift of 5 degrees.

\section{REFERENCES}

[1] P. Hasler, B.A. Minch, C. Diorio, and C. Mead, "An autozeroing floating-gate amplifier", Circuits and Systems II: Digital and Analog Signal Processing, in Press

[2] P. Hasler, Foundations of Learning in Analog VLSI, California Institute of Technology, February 1997. Also at www.ee.gatech.edu/users/phasler.

[3] P.S. Churchland and T.J. Sejnowski, The Computational Brain, MIT Press, Cambridge, MA, 1992.

[4] Y. Tsividis, M. Banu, and J. Khaury, "Continuoustime MOSFET-C filters in VLSI," IEEE Transactions on Circuits and Systems, vol.33, no.2, 1986.

[5] S.T. Dupuie and M. Ismail, "High frequency CMOS transconductors," in C. Toumazou, F.J. Lidgey, and D.G. Haigh, Analogue IC Design: the Current-Mode Approach, Peter Peregrinus, London, 1990, pp. 181238 . 